\title{
Giant pleural fibrous tumor: a case report
}

\author{
Loreto Berjón de la Vega, Mariano García-Yuste, José-María Matilla \\ Department of Thoracic Surgery, University Clinic Hospital of Valladolid, Valladolid, Spain \\ Correspondence to: José-María Matilla. Department of Thoracic Surgery, University Clinic Hospital of Valladolid, Ramón y Cajal Avenue, 47005 \\ Valladolid, Spain. Email: jmatillag@saludcastillayleon.es.
}

\begin{abstract}
Pleural fibrous tumor (PFT) is an infrequent and indolent pleural tumor, and most patients remain asymptomatic for years. The diagnosis is usually incidental and in large-size cases, representing a major surgical challenge. They may be associated to paraneoplastic syndromes such as refractory hypoglycemia or Doege-Potter syndrome. The present case is a 50-year-old female who came with dyspnea, chronic cough and constitutional syndrome. The CT scan showed a large intrathoracic mass of $20 \times 18 \times 17 \mathrm{~cm}^{3}$ which compressed the right lower and middle lobes (RLL and RML). At the time of the operation, the approach was right lateral thoracotomy extended to the chondrocostal region. During the resection, the tumor was accidentally opened and at that time the patient showed hypoglycemic levels. It is not often a long size PFT with hypoglycemia at the moment of operation without previous diagnosis. PFT is usually asymptomatic and is diagnosed incidentally in half of the cases. Tumor resection is the treatment of choice, which also leads to the cure of paraneoplastic syndrome. For tumors larger than $5 \mathrm{~cm}$, thoracotomy is preferred, and both the tumor and its implant pedicle must be resected with safety margins.
\end{abstract}

Keywords: Pleural fibrous tumor (PFT); Doege-Potter syndrome; paraneoplastic syndrome; case report

Received: 15 October 2019; Accepted: 04 November 2019; Published: 19 December 2019.

doi: $10.21037 /$ ccts.2019.11.02

View this article at: http://dx.doi.org/10.21037/ccts.2019.11.02

\section{Introduction}

Pleural fibrous tumor (PFT) is an infrequent (5\%) (1) and indolent pleural tumor, and most patients remain asymptomatic for years. The diagnosis is usually incidental, or by compressive symptoms, in large-size cases, representing a major surgical challenge. Even though PFT is usually encapsulated, yet it can compress or be intimately attached to adjacent structures.

On the other hand, they may be associated to paraneoplastic syndromes such as refractory hypoglycemia or Doege-Potter syndrome (2-4\%) (1). The patient did not present with previous paraneoplastic syndrome, but it did exceptionally manifest itself during surgical resection.

\section{Case presentation}

A 50-year-old female, smoker with a history of childhood rheumatic fever came with dyspnea, chronic cough and constitutional syndrome. The patient had no medical familiar history of interest for this pathology. Not relevant information in the physical examinations. The CT scan showed a large intrathoracic mass of $20 \times 18 \times 17 \mathrm{~cm}^{3}$ which compressed the right lower and middle lobes (RLL and RML). It surrounded and displaced the inferior vena cava, with a large contact surface with the right cardiac cavities and diaphragm without infiltration. This mass extended towards the mediastinum, surrounding the main right bronchus, carina and descending aorta and encompassing $180^{\circ}$ of its circumference, also without infiltration (Figure 1). Magnetic resonance imaging (MRI) showed a wide plane of contact with main vessels, heart, chest wall, diaphragm and spinal column, infiltration being ruled out.

The respiratory function was compromised by the large intrathoracic mass and the spirometry showed a restrictive pattern (FVC 1,490 cc 49\%, FEV1 1,060 cc $41 \%$, FEV1/FVC $84 \%)$. The involvement of the right 

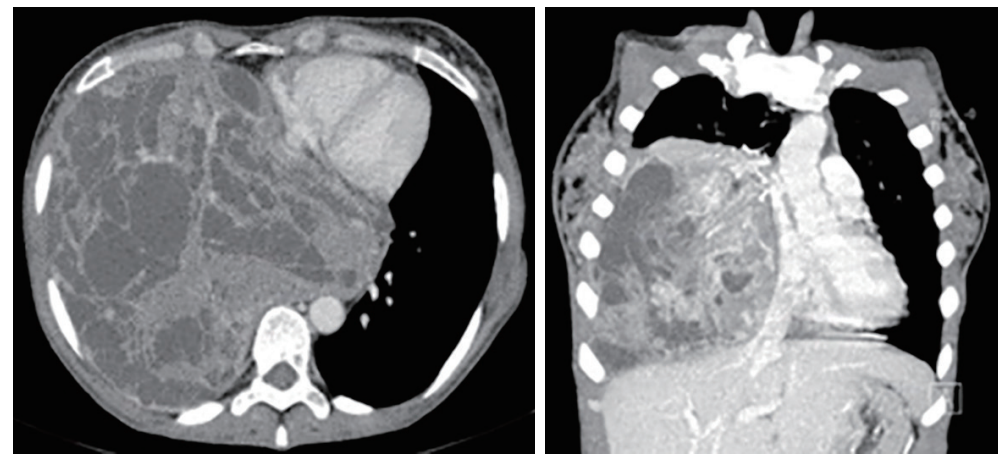

Figure 1 The CT images show the large size PFT toward the mediastinum and the desplacement of the cardiac structures.

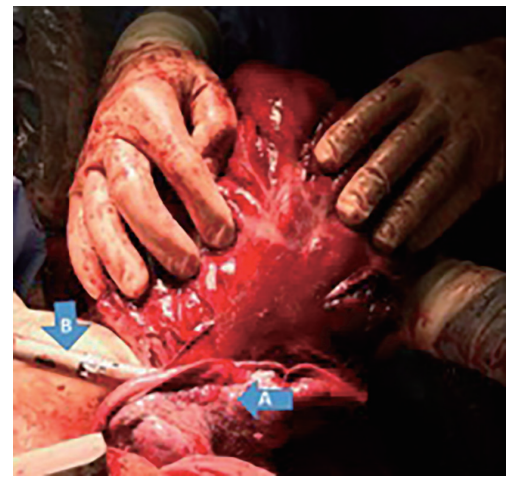

Figure 2 The main pedicle was removed by a RLL wedge resection (A) using endo-stapler (B). RLL, right lower lobe.

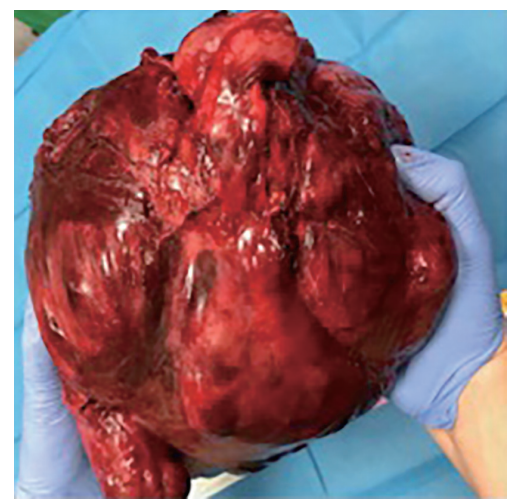

Figure 3 The large size PTF resected, outside the patient. PFT, pleural fibrous tumor.

cavities caused a moderate pulmonary hypertension (PSAP $45 \mathrm{mmHg}$ ) without compromising global systolic function (FEVI 60-65\%). The pulmonary perfusion scan showed heterogeneous distribution (left lung $81 \%$ ). The result of the transthoracic large needle biopsy was compatible with solitary fibrous tumor.

At the time of the operation, the patient was placed in $45^{\circ}$ right lateral decubitus as she did not tolerate complete lateral decubitus due to hemodynamic compromise. The approach was right lateral thoracotomy extended to the chondrocostal region. The tumor reached the base of the RLL, where the main pedicle was located, and it also had neoformed nutritional vessels from the parietal pleura. The resection was begun from the right costophrenic sinus and diaphragmatic base toward the anterior mediastinal region, the adhesions and peripheral nutrient vessels were released by using LigaSure ${ }^{\mathrm{TM}}$ and a vascular endo-stapler. Finally, the main pedicle was removed by means of an RLL wedge resection using endo-stapler (Figures 2,3). The phrenic nerve was included in the tumor capsule and it was accidentally sectioned. During the resection, the tumor was accidentally opened and at that time the patient showed hypoglycemic levels.

The anatomopathological result was a benign pleural solitary fibrous tumor, with some mitosis (2 mitosis/10 c.g.a.). Positive Ki-67<10\%. Tumor cells expressed vimentin, CD34, Bcl-2 and CD99; they did not express CK-AE1, AE2, PS100, Actin, DSM, calponin, calretinin, CD117, CD56.

In the postoperative evolution, the patient presented with post-surgical left lower lobe (LLL) atelectasis that was resolved by respiratory physiotherapy, as well as asymptomatic right hemidiaphragm elevation, good analgesic control and complete pulmonary re-expansion at hospital discharge. In the follow-up, the patient is currently disease-free (Figure 4). 


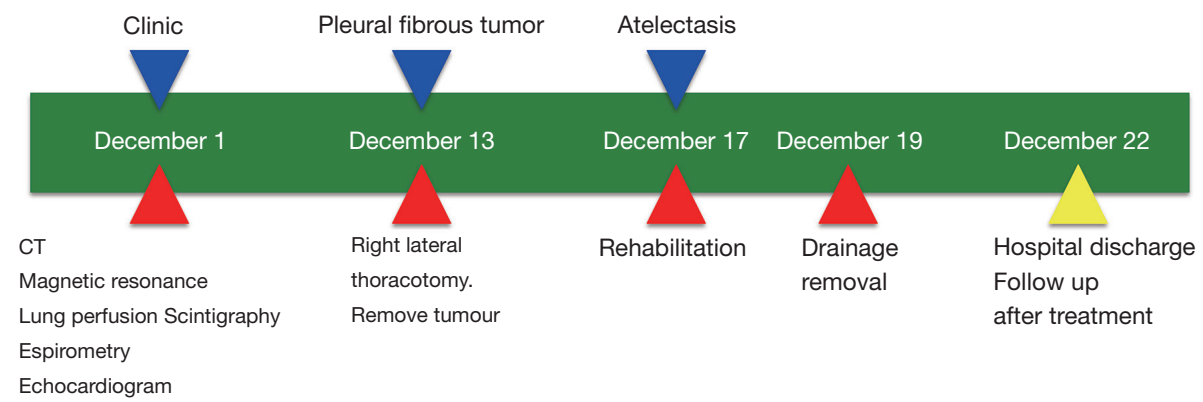

Figure 4 Timeline of giant pleural fibrous tumor.

\section{Discussion}

There are fewer than 2,000 cases described in the literature, most of them retrospective and from a single centre $(1,2)$. The distribution is homogeneous between both sexes, usually between 40 and 60 years old. Approximately half of the cases are diagnosed incidentally; the most frequent symptoms are chronic cough and dyspnea resulting from intrathoracic occupation, like the case reported. Chest pain is characteristic of those who depend on parietal pleura, especially if an infiltration of the latter coexists (3).

Doege-Potter syndrome was described in $1930(4,5)$ and it is characteristic of large tumors $(3,6)$, such as the case reported. Historically it was considered characteristic of malignant PFTs. However, in a relatively recent work Meng et al. (6) carried out a review of the literature from 1979 to 2011, without finding a clear association between this syndrome and malignant histology.

Hypoglycemia is the product of genetic disregulation in NAB2-STAT6 that leads to the overexpression of a molecule similar to IGF-2 (insulin growth factor type 2), known as "big IGF2" $(2,7)$, which can be found elevated in blood and inside tumor cells. It stimulates glucose consumption both in the body and in tumor cells, and also directly inhibits the mechanism of glucose synthesis in pancreatic cells, hepatic cells and fatty tissue. Clinical hypoglycemia improves with tumor resection (8). In our case, hypoglycemia only manifested itself at the time of tumor resection, as preoperatively the patient maintained glycemic levels within the normal range.

PFT can depend on either parietal (20\%) or visceral $(80 \%)$ pleura (1). The latter are frequently pediculated and it is in this implantation pedicle where the main nutritious artery is lodged. To achieve a complete resection in pediculated pulmonary fibrous tumors (PFTs), wedge resection of the pulmonary parenchyma surrounding the base of implantation is generally recommended, which is performed by means of an endostapler, with margins of $1-2 \mathrm{~cm} \mathrm{(2).} \mathrm{There} \mathrm{is} \mathrm{no} \mathrm{evidence} \mathrm{to} \mathrm{recommend} \mathrm{lobectomy}$ over sublobar resection (2). Resection of the pedicle itself may not be sufficient and subsequent recurrence may be associated (9).

On the other hand, the vascular supply can be multifocal, mainly thoracic aorta or internal mammary artery. Cases depending on phrenic arteries, bronchial and even abdominal aorta have also been reported, and in these cases angiography is of crucial help. In addition, preoperative embolization has been shown to reduce intraoperative bleeding and allows safer resections (10). In our case, the main nutritional vascular supply depended on the pulmonary circulation of the RLL, so no preoperative embolization was indicated.

The choice of approach will mostly depend on the tumor size. Thus, a minimally invasive technique is generally recommended in tumors smaller than $5 \mathrm{~cm}$ $(1,11)$. The large size of the tumor $(20 \mathrm{~cm})$ made us opt for a lateral thoracotomy approach in order to obtain a good visualization of the surgical field and therefore achieve a safer complete resection.

Microscopically, these tumors are characterized by being a connective tissue matrix with fibroblasts arranged without an established pattern ("patternless pattern", as described by Stout) (12). Immunohistochemical characteristics help to differentiate it from mesothelioma or sarcoma $(1,3)$. PFT expressed CD34, vimentin, Bcl2, CD99, STAT-6. In addition, in our case, the low mitotic index ( $<2$ mitosis/ c.g.a.), the absence of necrosis or hemorrhage and Ki-67 $<10 \%$ support the diagnosis of benign PFT (3). 
The benign PFT prognosis is good. A 5-year survival rate of $90 \%$ and a 10 -year survival rate of around $86 \%$ is described (13). Recurrences are unusual. Still, some authors recommend long-term follow-up for at least 15 years (1). de Perrot et al. (8) established a classification according to intraoperative findings and anatomopathological characteristics in order to predict behavior and future recurrences. In our case, as it is a pediculated PFT without histological signs of malignancy, following this classification (8) it would be stage 0 , probability of recurrence $2 \%$. However, we will carry out a long-term follow-up by CT scan. The main limitation is that it is a unique rare case with a great size and paraneoplastic syndrome with intraoperative symptoms.

\section{Conclusions}

PFT is an infrequent tumor of mesenchymal lineage. It is usually asymptomatic and is diagnosed incidentally in half of the cases. In addition, it is sometimes associated to paraneoplastic syndromes such as refractory hypoglycemia, known as Doege-Potter syndrome, produced by the overexpression of "big IGF2". Tumor resection is the treatment of choice, which also leads to the cure of paraneoplastic syndrome. For tumors larger than $5 \mathrm{~cm}$, thoracotomy is preferred, and both the tumor and its implant pedicle must be resected with safety margins. Preoperative embolization prevents massive bleeding during resection, offering safer resection. Survival of benign PFT is high with minimal recurrence rates; however, long-term follow-up is required.

\section{Acknowledgments}

Funding: None.

\section{Footnote}

Conflicts of Interest: All authors have completed the ICMJE uniform disclosure form (available at http://dx.doi. org/10.21037/ccts.2019.11.02). The authors have no conflicts of interest to declare.

Ethical Statement: The authors are accountable for all aspects of the work in ensuring that questions related to the accuracy or integrity of any part of the work are appropriately investigated and resolved. Written informed consent was obtained from the patient for publication of this Case Report and any accompanying images. All efforts to remove patient health information and maintain anonymity were made.

Open Access Statement: This is an Open Access article distributed in accordance with the Creative Commons Attribution-NonCommercial-NoDerivs 4.0 International License (CC BY-NC-ND 4.0), which permits the noncommercial replication and distribution of the article with the strict proviso that no changes or edits are made and the original work is properly cited (including links to both the formal publication through the relevant DOI and the license). See: https://creativecommons.org/licenses/by-nc-nd/4.0/.

\section{References}

1. Cardillo G, Lococo F, Carleo F, et al. Solitary fibrous tumors of the pleura. Curr Opin Pulm Med 2012;18:339-46.

2. Sternbach JM, Yeldandi A, De Campo MM. Solitary fibrous tumors and other uncommon neoplasms of the pleura. In: Locicero J, Feins RH, Colson YC, Rocco G. editors. Shields' General Thoracic Surgery 2018;65:797-812.

3. England DM, Hochholzer L, McCarthy MJ. Localized benign and malignant fibrous tumors of the pleura. A clinicopathologic review of 223 cases. Am J Surg Pathol 1989;13:640-58.

4. Doege KW. Fibro-sarcoma of the mediastinum. Ann Surg 1930;92:955-60.

5. Potter RP. Intrathoracic tumors. Radiology 1930;14:60-2.

6. Meng W, Zhu HH, Li H, et al. Solitary fibrous tumors of the pleura with Doege-Potter syndrome: a case report and three-decade review of the literature. BMC Res Notes. BMC Res Notes 2014;7:515.

7. de Groot JW, Rikhof B, van Doorn J, et al. Non-islet cell tumour-induced hypoglycaemia: a review of the literature including two new cases. Endocr Relat Cancer 2007;14:979-93.

8. de Perrot M, Fischer S, Bründler MA, et al. Solitary fibrous tumors of the pleura. Ann Thorac Surg 2002;74:285-93.

9. Cardillo G, Carbone L, Carleo F, et al. Solitary fibrous tumors of the pleura: an analysis of 110 patients treated in a single institution. Ann Thorac Surg 2009;88:1632-7.

10. Pinedo JA, Robles E, Peña ES, et al. Tumor fibroso solitario gigante de la pleura. Cir Ciruj [Published 
January-February 2010]. Available online: https:// www.medigraphic.com/cgi-bin/new/resumen. cgi?IDARTICULO=25034

11. Liu J, Cai C, Wang D, et al. Video-assisted thoracoscopic surgery (VATS) for patients with solitary fibrous tumors of the pleura. J Thorac Oncol 2010;5:240-3.

doi: $10.21037 /$ ccts.2019.11.02

Cite this article as: Berjón de la Vega L, García-Yuste M, Matilla JM. Giant pleural fibrous tumor: a case report. Curr Chill Thorac Surg 2019;1:22.
12. Stout AP. Tumors of the pleura. Harlem Hosp Bull 1952;5:54-7.

13. Santos RS, Haddad R, Lima CE, et al. Patterns of recurrence and long-term survival after curative resection of localized fibrous tumors of the pleura. Clin Lung Cancer 2005;7:197-201. 